\title{
The role of inner border sign and ridge sign in detecting high-grade cervical intraepithelial neoplasia
}

\author{
Mihaela Grigore $^{1,2} *$, Camelia Cojocaru ${ }^{1}$, Sergiu Teleman ${ }^{3}$
}

\author{
${ }^{1}$ Medis Medical Centre, Iasi, Romania \\ ${ }^{2}$ Department of Obstetrics and Gynaecology, Grigore T. Popa University of Medicine and Pharmacy, Iasi, Romania \\ ${ }^{3}$ Department of Pathology, Grigore T. Popa University of Medicine and Pharmacy, Iasi, Romania
}

Received: 02 September 2016

Accepted: 028 September 2016

\author{
*Correspondence: \\ Dr. Mihaela Grigore, \\ E-mail: mihaela.grigore@edr.ro
}

Copyright: () the author(s), publisher and licensee Medip Academy. This is an open-access article distributed under the terms of the Creative Commons Attribution Non-Commercial License, which permits unrestricted non-commercial use, distribution, and reproduction in any medium, provided the original work is properly cited.

\begin{abstract}
Background: The objectives of the study were to evaluate the role of two pathognomonic colposcopic signs (inner border sign and ridge sign) in detecting high-grade cervical lesions.

Methods: A total of 122 patients with abnormal Pap smear who had colposcopy and biopsy or loop electrosurgical excision procedure were included. The correlations between the two signs pathognomonic signs (inner border, ridge sign) and pathological results were established. We also compared the two signs with Reid colposcopic index (RCI) in detecting high-grade cervical lesions.

Results: Both pathognomonic signs proved to have a good accuracy in detecting high-grade lesions of the cervix. The sensitivity, specificity, PPV and NPV for the inner border sign and ridge sign were: 15\%, 98\%, 92\%, 44\%, respectively, and $30 \%, 94 \%, 88 \%, 47 \%$, respectively. RCI has a sensitivity, specificity, positive predictive value (PPV) and negative predictive value (NPV) for predicting high-grade cervical intraepithelial lesions as follows: $86.3 \%, 83.6 \%, 88.7 \%$, and $80.3 \%$, respectively. The simultaneous presence of both signs increased the specificity and PPV to $100 \%$, while sensitivity and NPV were $4 \%$ and $55 \%$.

Conclusions: Pathognomonic signs - inner sign and ridge sign- have a good specificity in predicting high-grade cervical intraepithelial lesions, but they are present in only $9.8 \%$, respectively $20.5 \%$ of cases with high-grade cervical intraepithelial lesions.
\end{abstract}

Keywords: High-grade cervical lesions, Inner border sign, Ridge sign, Reid colposcopic index

\section{INTRODUCTION}

Cervical cancer is the second most common cause of cancer death in women worldwide. Each year, 270,000 women die from cervical cancer, and another 500,000 are newly diagnosed. ${ }^{1}$ Primary prevention of cervical cancer employs the human papillomavirus (HPV) vaccine, and secondary prevention is targeted at detecting preneoplastic lesions using screening techniques such as cytology or HPV testing. Patients with abnormal cytology or HPV results are further investigated using colposcopy and histology to detect high-grade cervical intraepithelial lesions (CINs). High-grade lesions are removed using excisional or ablative surgical techniques.
Although colposcopy is mandatory for evaluating abnormal Pap smears, colposcopic findings can be misleading. The subjectivity of the findings imposed the implementation of a scoring system. The most commonly used system is the Reid Colposcopic Index (RCI), which is a method of colposcopically grading the severity of premalignant cervical lesions and could be useful in predicting the histologic grade of cervical disease, readily permitting differentiation between low-grade and highgrade disease. ${ }^{2}$ Hence, the use of the RCI helps direct the clinician to perform a biopsy of the most significant abnormal cervical lesions and enhances the formulation of the colposcopic impression. ${ }^{3}$ The accuracy of the RCI in different studies varies. Some investigations have 
reported it to have a high accuracy, but in others, the accuracy and interobserver agreement were poor even for experienced colposcopists. ${ }^{4-6}$ The differences in the studies regarding the accuracy of RCI warrant using additional colposcopic signs to improve the method's accuracy. In 2011, the International Federation for Cervical Pathology and Colposcopy (IFCPC) revised the colposcopic terminology by adding two recently described pathognomonic criteria that are highly associated with the presence of high-grade CIN, the inner border sign and ridge sign. ${ }^{7}$

This article evaluates the value of the pathognomonic signs in detecting high-grade cervical lesions.

\section{METHODS}

This is a prospective analytical comparative study. We analysed all patients referred to our colposcopy service and who had a biopsy or excisional procedure (loop electrosurgical excision procedure (LEEP) or conization) during two years. The following patients were excluded from the study: patients who had colposcopy but who did not have biopsy or LEEP and no final pathological report, patients with transformation zone (TZ) type 3 (according to IFCPC) and patients whose pathological report showed micro-invasive or invasive cervical cancer. Thus, from the total number of patients initially referred for colposcopy, 122 patients were eligible for inclusion.

The indication for colposcopic examination was the abnormal Pap test result. All the colposcopic examinations had reports recorded. By colposcopy, the RCI was established before biopsy. The RCI considers four colposcopy signs: lesion margin, color of acetowhitening, vessels, and iodine staining. Each sign is assigned a score between 0 and 2 reflecting variation in colposcopic appearances. The index is the sum of all four characteristics. A score of $0-2$ indicates the lesion is suggestive of CIN I, between 3-5 is suggestive for CIN I or II and an index of 6-8 suggests high-grade CIN (CIN II or III). During colposcopic examinations, we also looked for the presence of two pathognomonic signs (inner border and ridge sign) at the level of the TZ. The inner border sign is a sharp demarcation between a thin and dense aceto-white area within the same lesion, also called "lesion in a lesion" (Figure 1). ${ }^{8}$ The ridge sign is an opaque protuberance within the white epithelium directly adjacent to the squamocolumnar junction and resembles a mountain ridge (Figure 2). ${ }^{9}$ The TZ was evaluated according to IFCPC criteria: TZ type 1 is completely ectocervical and fully visible, type $2 \mathrm{TZ}$ has an endocervical component and type $3 \mathrm{TZ}$ has an endocervical component that is not fully visible. ${ }^{7} \mathrm{We}$ compared RCI scores and the presence of the pathognomonic signs with the pathological result. We estimated sensitivity and specificity, positive predictive value (PPV), negative predictive value (NPV), negative likelihood ratio (NLR) and positive likelihood ratio (PLR) for each pathognomonic sign. All statistical calculations were done using computer programs Microsoft Excel version 7 (Microsoft Corporation, NY, USA).

All patients give their written consent for colposcopic examination, biopsy, LEEP or conization. The study was approved by the internal review board.

\section{RESULTS}

A total of 122 patients were included in the study. The indications for colposcopy are listed (Table 1). The mean age ( \pm standard deviation) was $36,3+/-7,2$ years; $3.27 \%$ patients were younger than 25 years, $34.4 \%$ were between $25-35$ years and $62.3 \%$ were older than 35 years.

Table 1: Indications for colposcopy for cases who had a biopsy or LEEP.

\begin{tabular}{|c|c|c|}
\hline Pap test result & Cases & Percentage \\
\hline ASCUS $^{1}$ & 28 & $22.9 \%$ \\
\hline $\mathrm{ASC}-\mathrm{H}^{2}$ & 29 & $23.8 \%$ \\
\hline LSIL $^{3}$ & 30 & $24.6 \%$ \\
\hline $\mathrm{HSIL}^{4}$ & 35 & $28.7 \%$ \\
\hline Total cases & 122 & $100 \%$ \\
\hline \multicolumn{3}{|c|}{$\begin{array}{l}{ }^{1} \text { ASCUS: Atypical Squamous Cells of } \\
\text { Significance; }{ }^{2} \text { ASC-H: Atypical Squamous Cells Cannot } \\
\text { exclude High-grade squamous intraepithelial lesion; }{ }^{3} \text { LSIL: } \\
\text { Low-grade squamous intraepithelial lesion; }{ }^{4} \text { HSIL: High-grade } \\
\text { squamous intraepithelial lesion. }\end{array}$} \\
\hline
\end{tabular}

Table 2: Indications for colposcopy for cases who had a biopsy or LEEP.

\begin{tabular}{|llllll|}
\hline $\begin{array}{l}\text { Cell } \\
\text { abnormality }\end{array}$ & $\begin{array}{l}\text { Normal/ } \\
\text { benign } \\
\text { findings }\end{array}$ & CINI & $\begin{array}{l}\text { CIN } \\
\text { II- III }\end{array}$ & CIS & Total \\
\hline ASCUS & 4 & 14 & 10 & 0 & 28 \\
\hline ASC-H & 0 & 7 & 17 & 5 & 29 \\
\hline LSIL & 2 & 20 & 8 & 0 & 30 \\
\hline HSIL & 1 & 1 & 24 & 9 & 35 \\
\hline & 7 & 42 & 60 & 13 & 122 \\
\hline
\end{tabular}

Table 3: Correlation between RCI and pathological report.

\begin{tabular}{|lllll|}
\hline & $\begin{array}{l}\text { Score } \\
0-2\end{array}$ & $\begin{array}{l}\text { Score } \\
3-5\end{array}$ & $\begin{array}{l}\text { Score } \\
6-8\end{array}$ & Total \\
\hline $\begin{array}{l}\text { Normal } \\
\text { findings }\end{array}$ & 5 & 2 & 0 & 7 \\
\hline CIN I & 5 & 29 & 8 & 42 \\
\hline $\begin{array}{l}\text { CIN II- } \\
\text { III, CIS }\end{array}$ & 0 & 10 & 63 & 73 \\
\hline Total & 10 & 41 & 71 & 122 \\
\hline
\end{tabular}

A single biopsy was performed in 24 patients (19.6\%), two biopsies were performed in 40 patients $(32.7 \%)$ and 58 patients $(47.5 \%)$ underwent LEEP or conization. The pathological report was: benign cervical conditions, 7 cases (5.73\%); CIN I, 42 cases (34.42\%); high-grade lesions (CIN II or III), 60 cases (49.18\%); and carcinoma 
in situ (CIS), 13 cases (10.65\%). In patients where biopsy confirmed a high-grade lesion, a LEEP or conization was performed thereafter. We calculated the correlation between Pap test and final histologic diagnosis (Table 2). In patients younger than 25 years, the histology was as follows: CIN I, 3 cases; and high-grade CIN, 1 case. In women between 25 and 34 years old, $32.5 \%$ had normal histology, $48.8 \%$ had CIN I, and $18.6 \%$ had high-grade CIN/CIS. In women older than 35 years old, $9.3 \%$ had normal histology, 33.3\% had CIN I, and $57.3 \%$ had highgrade CIN/CIS.

Table 4: Prevalence of pathognomonic signs correlated with histologic diagnosis.

\begin{tabular}{|lll|}
\hline $\begin{array}{l}\text { Normal } \\
\text { findings }\end{array}$ & 0 & Ridge \\
\hline CIN I & 1 & 0 \\
\hline $\begin{array}{l}\text { CIN II-III, } \\
\text { CIS }\end{array}$ & 11 & 3 \\
\hline Total & 12 & 22 \\
\hline
\end{tabular}

The RCI was established for each case, and the correlation between Reid score and final pathological report is presented in Table 3 . The sensitivity, specificity, PPV and NPV for RCI in detecting CIN I were: $77.2 \%$, $88.7 \%, 80.9 \%, 86.3 \%$, respectively, and for CIN III were: $86.3 \%, 83.6 \%, 88.7 \%, 80.3 \%$, respectively.

Table 5: Sensitivity, specificity, PPV and NPV for pathognomonic signs.

\begin{tabular}{|llccc|}
\hline CIN II-III & Sensitivity & Specificity & PPV & NPV \\
\hline $\begin{array}{l}\text { Inner border } \\
\text { sign }\end{array}$ & 15 & 98 & 92 & 44 \\
\hline Ridge sign & 30 & 94 & 88 & 47 \\
\hline $\begin{array}{l}\text { Both signs } \\
\text { simultaneously }\end{array}$ & 4 & 100 & 100 & 55 \\
\hline
\end{tabular}

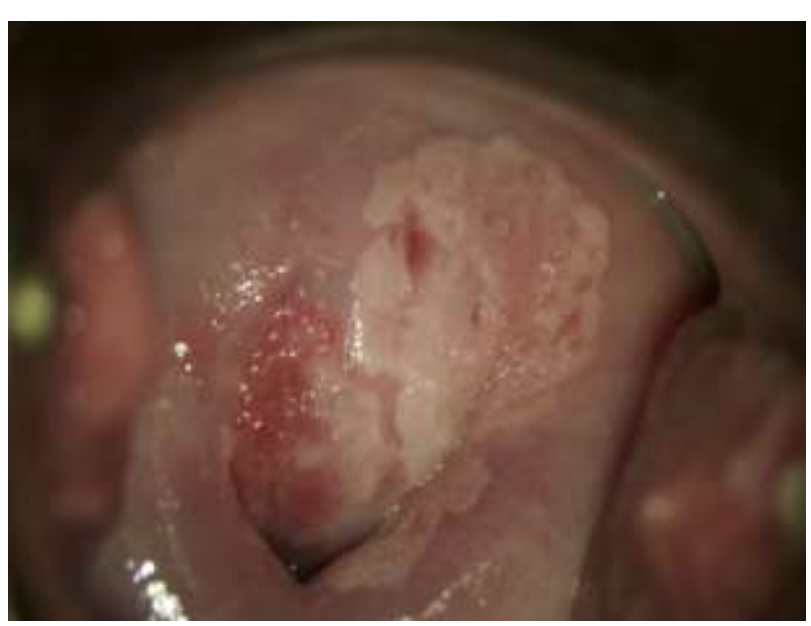

Figure 1: Inner border sign.

The inner sign was present in 12 cases $(9.8 \%)$, the ridge sign was present in 25 cases $(20.5 \%)$. Both signs were present in 3 cases with CIN III (2.5\%). The concordance between pathognomonic signs and histology is presented in Table 4 . The inner border sign was present in 12 cases $(9.8 \%)$. Of 73 patients with high-grade lesion or CIS, 11 had the inner border sign in colposcopy. The sensitivity, specificity, PPV, and NPV of the inner border sign in detecting high-grade CIN were 15\%, 98\%, 92\%, 44\%, respectively (Table 5).

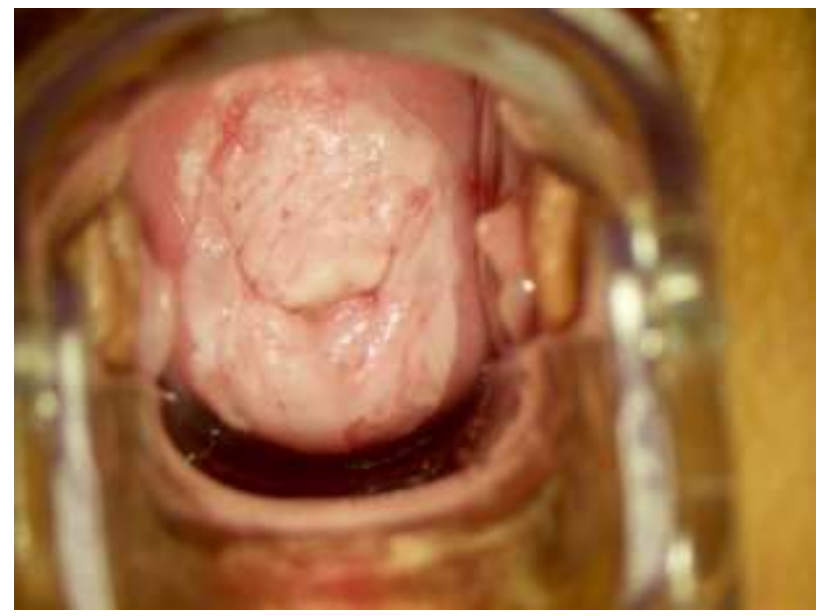

Figure 2: Ridge sign.

The ridge sign was present in 25 patients (20.5\%). Of 73 patients with high-grade lesion or CIS, 22 had the ridge sign during colposcopy. Sensitivity, specificity, PPV, and NPV of the inner border sign in detecting high-grade CIN were $30 \%, 94 \%, 88 \%, 47 \%$, respectively (Table 5).

\section{DISCUSSION}

Cervical cancer is a public health burden, particularly in developing countries. In well-developed countries, the incidence of cervical cancer has decreased because of cervical cytology screening. Because of the relatively low specificity of cytology, not all patients with cellular abnormalities will need treatment. Current approaches to cervical cancer prevention interpose colposcopy as a triage test to better define which women need treatment, and one important goal of colposcopy is to detect highgrade cervical lesions. ${ }^{5}$

According to the European Federation of Colposcopy (EFC), differentiating between low- and high-grade lesions is one of the quality indicators for colposcopy practice. ${ }^{10}$ EFC recommends that the percentage of highgrade lesions in LEEP and conization should be at least $85 \%$. This percentage ensures the unnecessary treatment of the cervix. Knowing the natural history of cervical lesions and to reduce the number of unnecessary interventions on the uterine cervix, especially if a seeand-treat management is followed, it is important to predict the high-grade lesions with the aid of colposcopy. The problem that arises is the subjective nature of colposcopy that may lead to differences in interpretation of the colposcopic findings. In 1985, Richard Reid introduced a colposcopic index to differentiate low-grade 
cervical disease from high-grade disease. This score may assist the beginner in assessing the characteristics of the abnormal TZ. As with any grading system, some subjectivity exists in the scoring of each colposcopic sign, particularly for color.

Our study found RCI had good sensitivity and specificity in detecting both low-grade CIN and high-grade CIN (77.2\%, 88.7\%, and $86.3 \%, 83.6 \%$, respectively). Thus, RCI can accurately predict histology. The obtained results could be explained by the more than $10-15$ years' coloposcopy experience of the gynaecologists involved in our study. In addition, colposcopy was not blinded for Pap smear results or other factors (HPV results, cervical pathology in antecedents, or history of smoking). In the literature, RCI proved also to be a good tool even for residents with little experience in colposcopy. ${ }^{11}$ In contrast, some of the recent literature has discredited the $\mathrm{RCI}$ as inaccurate and non-reproducible. Ferris showed in the ASCUS/LSIL Triage Study for Cervical Cancer (ALTS) trial that colposcopists using the RCI failed to detect CIN II/III at the levels expected. ${ }^{3}$ According to Massad, clinical colposcopic impression and the modified Reid Index components that contribute to it do not discriminate between aceto-white lesions that harbor CIN II+ and those that do not. ${ }^{5}$

Because of the contradictory findings regarding RCI, in recent years some pathognomonic signs were studied to evaluate their specificity and sensitivity for detecting high-grade lesions. Both "inner border sign" and "ridge sign" were introduced in the last colposcopy nomenclature (IFCPC 2011) as major lesions because of their significant validity as markers of high-grade CIN.

Scheungraber et al studied the correlation between the inner border sign and CIN II or III and its association with specific human papillomavirus (HPV) types and the patient's age. The occurrence of the inner border sign was evaluated retrospectively by two independent colposcopists on 947 women referred for an abnormal cervical finding. The prevalence of the inner border sign in women with an atypical $\mathrm{TZ}$ was $7.6 \%$. In the same study, in $70 \%$ of women with the inner border sign, CIN II or III was confirmed histologically. In patients with the inner border sign, the odds ratio for CIN II or III was 7.7 (95\% CI=4.2-14.3). There was no significant association between the inner border sign and any high-risk HPV type. The authors concluded that the inner border sign is a rare colposcopic phenomenon, but is highly specific for CIN II or III in young women.

In another study, Vercellino et al examined the association between three pathognomonic criteria: inner border, ridge sign, and rag sign, and high-grade CIN on 335 patients referred for colposcopy and biopsy/LEEP. ${ }^{12}$ Sensitivity, specificity, PPV, and NPV of the inner border in detecting high-grade CIN were $20 \%, 99 \%, 97.9 \%$, and $34.8 \%$ respectively. The positive likelihood ratio $(\mathrm{LR}+)$ was 20.3 and the negative likelihood ratio (LR-) was
0.81. Our study found similar results. The sensitivity, specificity, PPV, NPV in our study were $15 \%, 98 \%, 92 \%$, $44 \%$, respectively, so we can say that the inner border represents an important sign in detecting high-grade CIN.

Scheungraber et al studied the other pathognomonic sign, the ridge sign, by retrospectively analyzing records of 592 patients referred for punch or cone biopsy. ${ }^{9}$ The sensitivity of the ridge sign for detecting CIN II or III was $33.1 \%$; and specificity was $93.1 \%$. Women with the ridge sign were significantly younger than women with no ridge sign $(\mathrm{p}<0.001)$. The ridge sign was associated with the presence of HPV $16(p<0.001)$. The ridge sign is a highly specific marker for CIN II or III and is associated with HPV 16 and young age.

Vercellino et al reported the ridge sign sensitivity, specificity, PPV and NPV in detecting high-grade CIN as $52.5 \%, 96.4 \%, 96.8 \%$, and $46.6 \%$, respectively. ${ }^{12}$ The $\mathrm{LR}+$ ratio was 13.2 , and the LR- ratio was 0.49 . In our study, ridge signs were present in 25 cases $(20.49 \%)$, from which 3 patients had CIN I and 23 patients had CIN II, II or CIS. The sensitivity, specificity, PPV and NPV in our study were $30 \%, 94 \%, 88 \%, 47 \%$, respectively.

In our study, both RCI and the pathognomonic signs (inner border and ridge sign) proved to have high sensitivity and specificity in detecting high-grade lesions of the uterine cervix. The inner border and ridge sign are objective and effective colposcopic signs and are significantly associated with high-grade CIN. The problem is that they are simply present or absent: their presence is highly suggestive for high-grade lesions, but their absence does not necessarily mean absence of a high-grade CIN. In cases where the pathognomonic signs are absent, RCI is still a valuable tool that should be used for grading CIN.

Our study has some limitations. First, the study focused on only two pathognomonic signs, and in recent years, additional signs have been described (e.g., rag sign, cuffed gland openings). Also, the results of cytology were known before colposcopy examination. It would be interesting to establish if there are any correlations between HPV and the parameters studied, but because the HPV test is not free in our country, only a small number of patients were tested.

More studies are needed in the future to validate pathognomonic signs as parameters for high-grade cervical lesions. In addition, it is important to make correlations between HPV and each sign or to study if the presence of these signs in low-grade lesions could indicate the progression of the lesion to a more severe lesion.

\section{CONCLUSION}

Colposcopy should be considered as a routine technique in daily practice, but the contradictory results from the 
literature regarding its accuracy suggests that its technique has yet to be improved. Pathognomonic signs inner sign and ridge sign- have a good specificity in predicting high-grade cervical intraepithelial lesions, but unfortunately they are present in only $9.8 \%$, respectively $20.5 \%$ of cases with high-grade cervical intraepithelial lesions.

Funding: No funding sources

Conflict of interest: None declared

Ethical approval: The study was approved by the Institutional Ethics Committee

\section{REFERENCES}

1. Arbyn M, Castellsagué X, de Sanjosé S, Bruni L, Saraiya M, Bray, et al. Worldwide burden of cervical cancer in 2008. Ann Oncol. 2011;22:2675-86.

2. Reid R, Stanhope CR, Herschman BR, Crum CP, Agronow SJ. Genital warts and cervical cancer. IV. A colposcopic index for differentiating subclinical papillomaviral infection from cervical intraepithelial neoplasia. Am J Obstet Gynecol. 1984;149:815-23.

3. Ferris DG, Litaker MS, ALTS Group. Prediction of cervical histologic results using an abbreviated Reid Colposcopic Index during ALTS Am J Obstet Gynecol. 2006;194:704-10.

4. Mousavi AS, Fakour F, Gilani MM, Behtash N, Ghaemmaghami F, Karimi, et al. A prospective study to evaluate the correlation between Reid colposcopic index impression and biopsy histology. J Low Genit Tract Dis. 2007;11:147-50.

5. Massad LS, Jeronimo J, Katki HA, Schiffman M. National Institutes of Health/American Society for Colposcopy and Cervical Pathology Research Group. The accuracy of colposcopic grading for detection of high-grade cervical intraepithelial neoplasia. J Low Genit Tract Dis. 2009;13:137-44.

6. Ferris DG, Litaker M. ALTS Group. Interobserver agreement for colposcopy quality control using digitized colposcopic images during the ALTS trial. J Low Genit Tract Dis. 2005;9:29-35.

7. Bornstein J, Bentley J, Bösze P, Girardi F, Haefner $\mathrm{H}$, Menton M, et al. 2011 colposcopic terminology of the International Federation for Cervical Pathology and Colposcopy. Obstet Gynecol. 2012;120:166-72.

8. Scheungraber C, Glutig K, Fechtel B, Kuehne-Heid R, Duerst M, Schneider A. Inner border, a specific and significant colposcopic sign for moderate or severe dysplasia (cervical intraepithelial neoplasia 2 or 3). J Low Genit Tract Dis. 2009;13:1-4.

9. Scheungraber C, Koenig U, Fechtel B, Kuehne-Heid R, Duerst M, Schneider A. The colposcopic feature ridge sign is associated with the presence of cervical intraepithelial neoplasia $2 / 3$ and human papillomavirus 16 in young women. J Low Genit Tract Dis. 2009;13:13-6.

10. Moss EL, Arbyn M, Dollery E, Leeson S, Petry KU, Nieminen $\mathrm{P}$ et al. European Federation of Colposcopy quality standards Delphi consultation. Eur J Obstet Gynecol Reprod Biol. 2013;170:255-8.

11. Shojaei H, Yarandi F, Ghozati L, Yarandi N, IzadiMood N, Eftekhar Z. Acceptable predictive accuracy of histopathology results by colposcopy done by Gynecology residents using Reid index. Arch Gynecol Obstet. 2013;287:345-9.

12. Vercellino GF, Erdemoglu E, Chiantera V, Vasiljeva K, Drechsler I, Cichon G, et al. Validity of the colposcopic criteria inner border sign, ridge sign, and rag sign for detection of high-grade cervical intraepithelial neoplasia. Obstet Gynecol. 2013;121:624-31.

Cite this article as: Grigore M, Cojocaru C, Teleman S. The role of inner border sign and ridge sign in detecting high-grade cervical intraepithelial neoplasia. Int J Reprod Contracept Obstet Gynecol 2016;5:3717-21. 